\title{
Pleuroparenchymal fibroelastosis in Korean patients: clinico-radiologic-pathologic features and 2-year follow-up
}

\author{
Jae Ha Lee ${ }^{1}$, Eun Jin Chae ${ }^{2}$, Joon Seon Song 3 , Miae Kim', and Jin Woo Song ${ }^{4}$
}

\begin{abstract}
${ }^{1}$ Division of Pulmonology and Critical Care Medicine, Department of Internal Medicine, Inje University Haeundae Paik Hospital, Busan; Departments of ${ }^{2}$ Radiology, ${ }^{3}$ Pathology, and ${ }^{4}$ Pulmonary and Critical Care Medicine, Asan Medical Center, University of Ulsan College of Medicine, Seoul, Korea
\end{abstract}

Received: September 8, 2019

Revised : October 25, 2019

Accepted: December 17, 2019

\section{Correspondence to}

Jin Woo Song, M.D.

Department of Pulmonary and

Critical Care Medicine, Asan

Medical Center, University of

Ulsan College of Medicine, 88

Olympic-ro 43-gil, Songpa-gu,

Seoul 05505, Korea

Tel: +82-2-3010-3993

Fax: +82-2-3010-6968

E-mail:jwsongasan@gmail.com

https://orcid.org/0000-0001-

5121-3522
Background/Aims: Pleuroparenchymal fibroelastosis (PPFE) is a type of rare idiopathic interstitial pneumonia that is characterized by predominantly upper lobe involvement with pleural fibrosis and subjacent parenchymal fibrosis. This study aimed to determine the clinico-radiologic-pathologic features and prognosis of Korean patients with PPFE.

Methods: A total of 26 patients who were confirmed to have PPFE by lung biopsy, were included, and their clinico-radiologic-pathologic findings were retrospectively analyzed.

Results: The mean follow-up period was 23.8 months, and the mean age of the patients was 62.5 years; $61.5 \%$ were men and $50 \%$ were smokers. Cough and dyspnea were the most frequent presenting symptoms, and restrictive pattern was the most common observation in lung function. In $84.6 \%$ of the subjects, lower lobe involvement was found on chest computed tomography, and the usual interstitial pneumonia (UIP) pattern was the most common (59.1\%). Among patients whose lower lobe was biopsied $(\mathrm{n}=13)$, the UIP pattern was the most common $(46.2 \%)$. Patients with lower lobe involvement were older and walked a shorter distance during the 6-minute walk test, compared to those without. Spontaneous pneumothorax was a common complication (26.9\%), and $15.4 \%$ of the patients died mostly due to pneumonia (100\%). The 1- and 3-year survival rates were $90.2 \%$ and $84.5 \%$, respectively.

Conclusions: Clinical features of Korean patients with PPFE were similar to those reported previously; however, lower lobe involvement was more frequent. During follow-up, one-fourth of the patients experienced pneumothorax and one-fifth died from pneumonia.

Keywords: Idiopathic interstitial pneumonias; Pulmonary fibrosis; Pleural diseases; Survival analysis

\section{INTRODUCTION}

Pleuroparenchymal fibroelastosis (PPFE) is a rare type of idiopathic interstitial pneumonia (IIP) [1], and was first reported in 2004 by Frankel et al. [2]. Pathological find- ings for PPFE include pleural fibrosis with subjacent intra-alveolar fibrosis and alveolar septal elastosis, while radiological findings include pleural thickening of the upper lobe, with subpleural fibrosis, on high-resolution computed tomography (HRCT) [3,4]. PPFE is known to 
predominantly involve the upper lobes; however, there is increasing evidence for its coexistence with interstitial lung disease (ILD) in the lower lobe ( $43 \%$ to $89 \%$ ), with the usual interstitial pneumonia (UIP) pattern being the most common [3-5]. PPFE shows diverse clinical courses, ranging from slow to rapid progression $[6,7]$. Previous studies have reported that the median survival period for patients with PPFE ranges from 35.3 months to 11 years, which is similar to that of patients with other IIPs [6,8-11].

Recently, Cha et al. [12] reported clinico-pathologic features of seven Korean patients with histologically proven PPFE. In this study, all patients showed a restrictive pattern in lung function and had underlying clinical conditions (four connective tissue disease, three organ transplantation, and one fungal infection). In five patients (71.4\%), lower lobe involvement was observed on HRCT. The UIP pattern (40\%) was the most common pathological finding in the lower lobe [12]. However, these results are limited by a small number of cases and the absence of follow-up data. In addition, it is still uncertain whether lower lobe involvement impacts the prognosis of PPFE patients. Therefore, the aim of this study was to evaluate the clinico-radiologic-pathologic features and clinical course in a larger number of Korean patients with PPFE, and to evaluate the clinical differences between patients with and without lower lobe involvement.

\section{METHODS}

\section{Study population}

All patients with PPFE, diagnosed from August 2010 to October 2017 at Asan Medical Center, Seoul, Republic of Korea, were included in this study. The diagnostic criteria for PPFE, based on the criteria suggested by Reddy et al. [3], were defined as follows: (1) dense subpleural airspace consolidation with traction bronchiectasis located predominantly in the upper lobe on HRCT and (2) histopathologic findings of subpleural intra-alveolar collagenous fibrosis and alveolar wall elastosis associated with collapsed alveoli, with or without collagenous thickening of the visceral pleura. The final diagnosis was established through a multidisciplinary discussion. The study protocol was approved by the Institutional Review Board of Asan
Medical Center (approval number: 2017-1656), and the requirement for written informed consent was waived due to the retrospective nature of this study.

\section{Clinical information}

Clinical and survival data for all patients were obtained from medical records, telephone interviews, and/or the records of the National Health Insurance of Korea. Spirometry, measurement of diffusing capacity of the lung for carbon monoxide (DLco), and plethysmography for the measurement of total lung capacity (TLC) were performed according to the recommendations of the American Thoracic Society (ATS)/European Respiratory Society (ERS) [13-15]. The results were expressed as percentages of normal predicted values. The 6-minute walk test (6MWT) was performed according to ATS guidelines [16-18]. Data from the follow-up clinic visits, which were conducted every 3 to 6 months, and those from hospitalization were reviewed to determine the changes in the lung function and development of complications.

\section{Radiologic-pathologic assessments}

HRCT scans were reviewed by one chest radiologist (E.J.C.) with 17 years of experience, who was blinded to the clinical and pathologic information of patients. Upper and lower zones were divided at the carina level, and the cephalocaudal distribution (upper lobe only, upper and lower lobes) was evaluated. In cases showing lower lobe involvement, radiologic criteria for PPFE were applied to the lower lobe, and other patterns not meeting the radiologic criteria for PPFE were classified according to the IIP classification [1]. Histopathologic features were assessed by one pathologist (J.S.S.), who was blinded to the clinical and radiologic information of patients. In the case of multiple lobe biopsy, the pathologic pattern of the lower lobe was also evaluated.

\section{Statistical analysis}

All values are expressed as the mean \pm standard deviation for continuous variables and as percentages for categorical variables. The Student's $t$ test or Mann-Whitney $U$ test was used for continuous data and the Pearson's chisquare test or Fisher's exact tests, for categorical data. Generalized linear mixed models were used for repeated measures of the pulmonary function test and 6MWT, 
Table 1. Baseline clinical characteristics of the patients $(n=26)$

\begin{tabular}{|c|c|}
\hline Characteristic & Value \\
\hline Age, yr & $62.5 \pm 9.9$ \\
\hline Male sex & $16(61.5)$ \\
\hline Ever smokers & $13(50.0)$ \\
\hline BMI, $\mathrm{kg} / \mathrm{m}^{2}$ & $20.1 \pm 2.9$ \\
\hline C-reactive protein, mg/dL & $0.42 \pm 0.95$ \\
\hline \multicolumn{2}{|l|}{ Symptoms at presentation } \\
\hline Cough & $18(69.2)$ \\
\hline Dyspnea & $21(80.8)$ \\
\hline Chest pain & $2(7 \cdot 7)$ \\
\hline Weight loss ${ }^{\mathrm{a}}$ & $6(23.1)$ \\
\hline No symptom & $1(3.8)$ \\
\hline \multicolumn{2}{|c|}{ Relevant underlying conditions } \\
\hline Organ transplantation ${ }^{\mathrm{b}}$ & $2(7.7)$ \\
\hline Chemotherapy & $3(11.5)$ \\
\hline Infection $^{c}$ & $11(42.3)$ \\
\hline No exposure & $11(42.3)$ \\
\hline
\end{tabular}

Values are presented as mean $\pm \mathrm{SD}$ or number (\%).

BMI, body mass index; CRP, C-reactive protein

${ }^{a}$ Weight loss: loss of body weight at least 10\% within 6 months or $5 \%$ in the last months.

${ }^{\mathrm{b}}$ Organ transplantation: 1 heart, 1 kidney.

${ }^{\mathrm{c}}$ Infection: 4 nontuberculous mycobacteria, 5 pulmonary tuberculosis, 2 fungal infection.

with time being the predictor variable. Fixed effects in all models were assessed against time, and an auto-regressive covariance matrix structure was utilized. The overall survival (OS) was estimated using Kaplan-Meier curves. Risk factors for mortality were analyzed using a Cox proportional hazard model with backward, stepwise elimination, and variables with $p<0.1$ in the univariate analysis were subjected to multivariate analysis. All $p$ values were two-tailed, with statistical significance set at $p<$ 0.05. All statistical analyses were performed using SPSS version 24.o (IBM Co., Armonk, NY, USA) and MedCalc 11.6.1.o statistical software (MedCalc Software bvba, Ostend, Belgium).

\section{RESULTS}

\section{Baseline clinical characteristics}

A total of 26 patients with PPFE were included in this
Table 2. Baseline physiological parameters and bronchoalveolar lavage fluid findings of the patients $(n=26)$

\begin{tabular}{|c|c|}
\hline Characteristic & Value \\
\hline \multicolumn{2}{|c|}{ Pulmonary function pattern } \\
\hline Restrictive pattern & $19(73.1)$ \\
\hline Normal & $6(23.1)$ \\
\hline \multicolumn{2}{|l|}{ Pulmonary function } \\
\hline FVC, \% predicted & $61.8 \pm 19.5$ \\
\hline DLco, $\%$ predicted & $55 \cdot 3 \pm 18.4$ \\
\hline TLC, \% predicted & $70.1 \pm 13.4$ \\
\hline RV, \% predicted & $69.5 \pm 19.2$ \\
\hline RV/TLC, \% & $38.3 \pm 9.4$ \\
\hline \multicolumn{2}{|l|}{ Six-minute walk test } \\
\hline Distance, meter & $445 \cdot 7 \pm 104 \cdot 7$ \\
\hline Initial $\mathrm{SpO}_{2}, \%$ & $97.0 \pm 1.3$ \\
\hline Lowest $\mathrm{SpO}_{2}, \%$ & $93.9 \pm 3.0$ \\
\hline \multicolumn{2}{|l|}{ Blood gas } \\
\hline $\mathrm{PaO}_{2}, \mathrm{mmHg}$ & $106.4 \pm 21.5$ \\
\hline $\mathrm{PaCO}_{2}, \mathrm{mmHg}$ & $37 \cdot 6 \pm 3.2$ \\
\hline RVSP, mmHg & $26.7 \pm 10.2$ \\
\hline \multicolumn{2}{|l|}{ Bronchoalveolar lavage } \\
\hline WBC count, cell/uL & $260.8 \pm 288.2$ \\
\hline Neutrophil, \% & $14 \cdot 5 \pm 17 \cdot 7$ \\
\hline Lymphocyte, \% & $16.5 \pm 15.0$ \\
\hline
\end{tabular}

Values are presented as number (\%) or mean $\pm \mathrm{SD}$.

FVC, forced vital capacity; DLco, diffusing capacity of the lung for carbon monoxide; TLC, total lung capacity; RV, residual volume; $\mathrm{SpO}_{2}$, saturation of peripheral oxygen; $\mathrm{PaO}_{2}$, partial pressure of oxygen; $\mathrm{PaCO}_{2}$, partial pressure of carbon dioxide; RVSP, right ventricular systolic pressure; WBC, white blood cell.

study. All diagnoses were confirmed by lung biopsy (15 surgical lung biopsy, 11 transbronchial lung biopsy). The mean follow-up period was 23.8 months. Among all patients, the mean age was 62.5 years (median, 63 [range, 43 to 78$]$ ), $61.5 \%$ were men, and 50\% were smokers (Table 1). Cough and dyspnea were the most frequently presented symptoms. Approximately $50 \%$ of the patients had a history of relevant underlying conditions and seven patients (26.9\%) had a history of spontaneous pneumothorax. One patient (3.8\%) had a family history of ILD (idiopathic pulmonary fibrosis [IPF]) within second-degree relatives. Most patients (73.1\%) revealed a mild restrictive ventilatory defect (Table 2). 

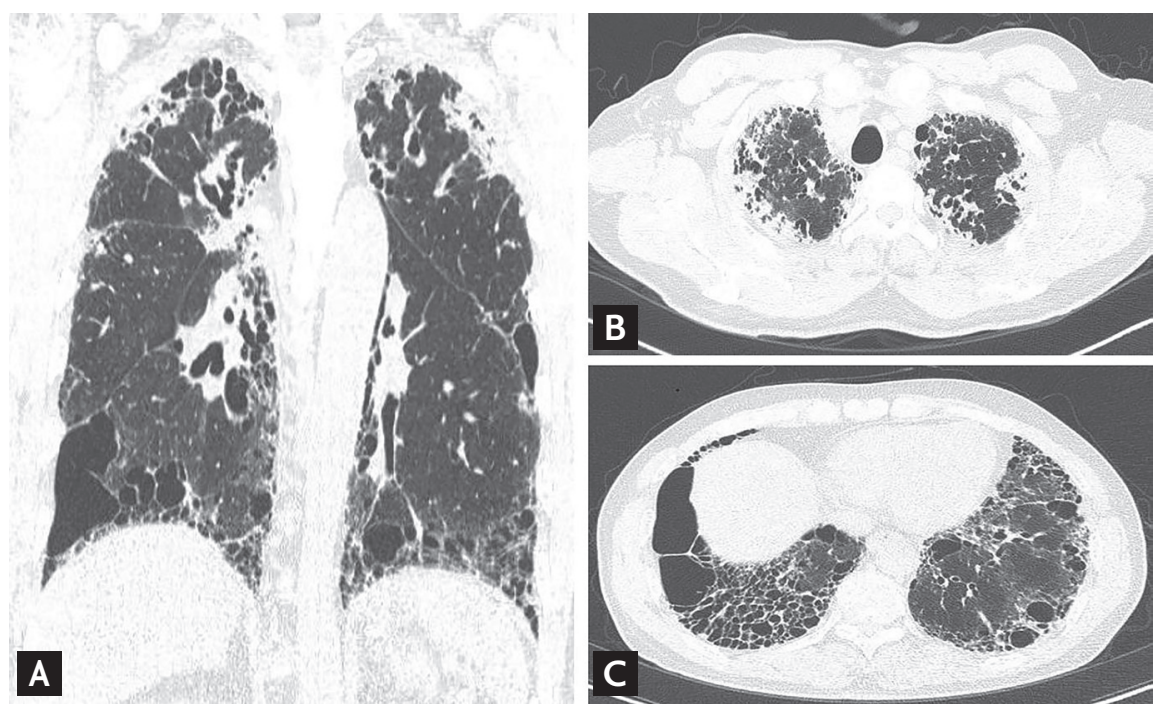

Figure 1. Representative chest computed tomography images of patients with pleuroparenchymal fibroelastosis (PPFE). Images of the patients show the PPFE pattern in the upper lung and the usual interstitial pneumonia (UIP) pattern in the lower lung. (A) Coronal computed tomography (CT) images demonstrate the PPFE pattern in the upper lobe and the UIP pattern in the lower (3) lobe. (B) Axial CT images show bilateral irregular-shaped subpleural nodular lesions, suggesting dense fibrosis in both upper lobes. (C) Axial CT image of the lower lung show areas of honeycombing cysts mixed with large emphysematous cysts, traction bronchiectasis, and coarse reticulation in both basal lungs, suggesting the UIP pattern.

Table 3. Radiological and pathological findings of the patients $(n=26)$

\begin{tabular}{lc}
\hline Characteristic & Value \\
\hline Distribution on HRCT & \\
Upper lobe alone & $4(15.4)$ \\
Upper and lower lobe & $22(84.6)$ \\
Radiological pattern (lower lobes) & 22 \\
\hline UIP pattern & $13(59.1)$ \\
\hline PPFE pattern & $8(36.4)$ \\
Unclassifiable & $1(4.5)$ \\
Pathologic findings (lower lobes) & 13 \\
\hline UIP pattern & $6(46.2)$ \\
OP pattern & $4(30.8)$ \\
PPFE pattern & $2(15.4)$ \\
Unclassifiable & $1(7.6)$ \\
\hline
\end{tabular}

Values are presented as number (\%).

HRCT, high-resolution computed tomography; UIP, usual interstitial pneumonia; PPFE, pleuroparenchymal fibroelastosis; OP, organizing pneumonia.

\section{Radiological and pathological findings}

All HRCT findings corresponded to PPFE based on radiological criteria (Fig. 1). Most patients (84.6\%) had coexistent involvement of ILD in their lower lobes on HRCT, and the UIP pattern was the most common (59.1\%), followed by PPFE (36.4\%), and unclassifiable pattern $(4.5 \%)$, respectively (Table 3$)$. In patients subjected to lower lobe biopsy $(n=13)$, the UIP pattern was the most common $(46.2 \%)$, followed by organizing pneumonia (30.8\%), PPFE (15.4\%), and unclassifiable pattern (7.6\%), respectively (Table 3). Elastic fiber (EF, 38.5\% of total subjects) was also found with other ILDs in the lower lobe.

\section{Clinical course and survival}

During follow-up, pneumothorax developed in seven patients (26.9\%), and lung function (forced vital capacity [FVC], DLco, TLC) and oxyhemoglobin saturation (initial and the lowest saturation of peripheral oxygen $\left[\mathrm{SpO}_{2}\right]$ during 6MWT) were significantly decreased (Fig. 2). Eighteen patients $(69.2 \%)$ were treated with steroids \pm immunosuppressants, and five (19.2\%) were treated with antifibrotic agents. Four patients (15.4\%) died during follow-up due to respiratory infection (100\%). The 1- and 3-year survival rates were $90.2 \%$ and $84.5 \%$, respectively (Fig. 3A). There were no differences in the 1- (100\% vs. $88.7 \%$ ) and 3-year survival rates (100\% vs. $81.8 \%$ ) between patients treated with antifibrotic agents and those without antifibrotic agents (log-rank test, $p=0.266)$. In a univariate Cox analysis, C-reactive protein, FVC, and distance during 6MWT were significant prognostic factors for mortality (Table 4). However, in multivariate Cox analysis, only FVC was found to be a significant factor affecting OS (hazard ratio [HR], 0.894; 95\% confidence interval [CI], 0.809 to $0.988 ; p=0.028$ ). 

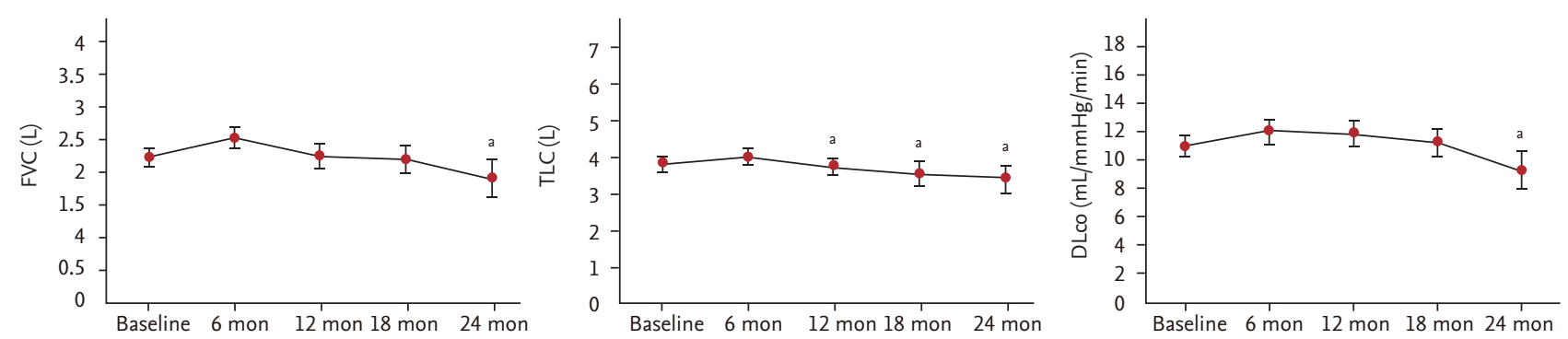

\section{A}

B

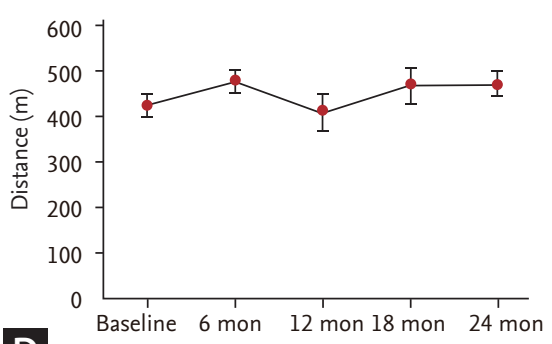

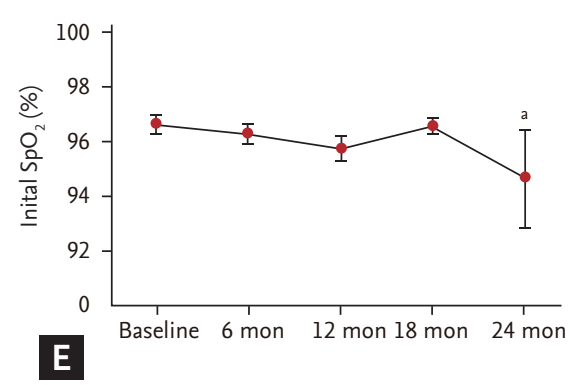

C

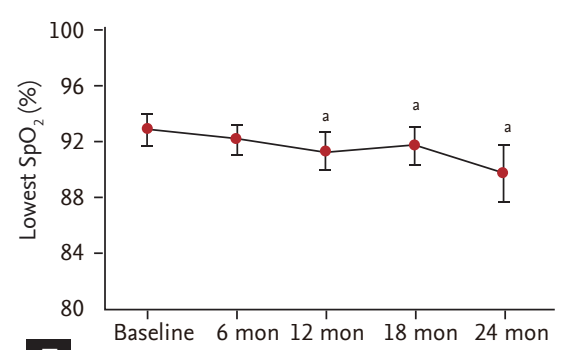

$\mathbf{F}$

Figure 2. Changes in lung function and exercise capacity over time in patients with pleuroparenchymal fibroelastosis. Changes over 24 months in (A) forced vital capacity (FVC), (B) total lung capacity (TLC), (C) diffusing capacity of the lung for carbon monoxide (DLco), and in (D) distance, (E) initial saturation of peripheral oxygen $\left(\mathrm{SpO}_{2}\right)$, and $(\mathrm{F})$, and the lowest $\mathrm{SpO} \mathrm{O}_{2}$ during 6-minute walk test. Each plot represents the mean \pm standard error of measurement of lung function or exercise capacity. ${ }^{\mathrm{a}} \mathrm{Sig}-$ nificant decrease in outcome variables compared to the baseline value.

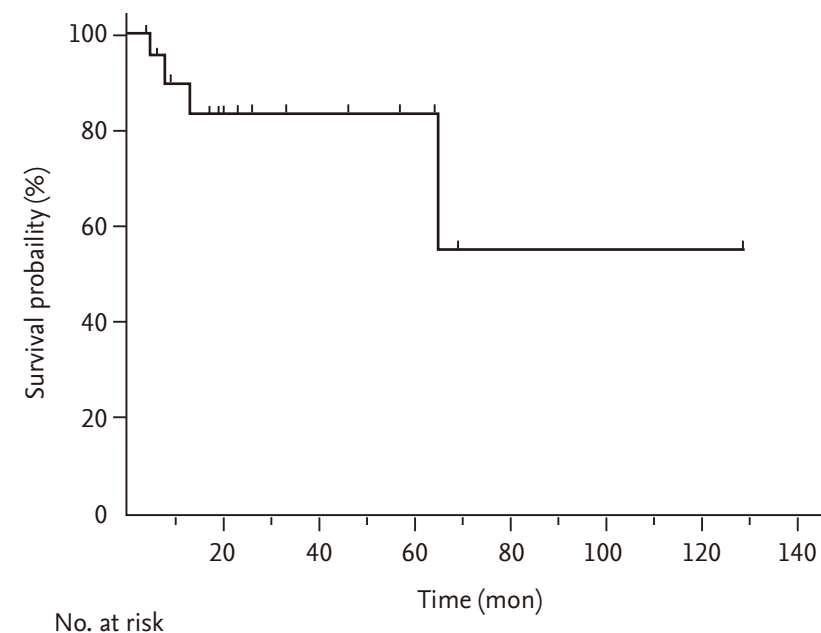

A 26 13 6 4 1 1

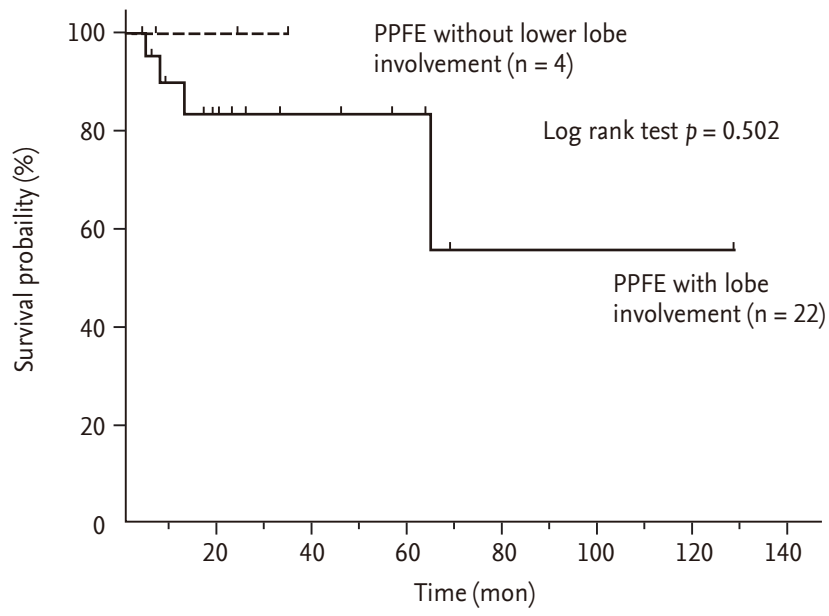

Figure 3. Kaplan-Meier survival curves in patients with pleuroparenchymal fibroelastosis (PPFE). (A) Survival curves of total patients with PPFE. (B) Comparison of survival curves between PPFE patients with and without lower lobe involvement.

\section{Comparison of clinical characteristics between PPFE patients with and without lower lobe involvement}

Among patients with PPFE, those with lower lobe involvement were older and had a shorter distance during
6MWT than those without (Table 5). However, development of pneumothorax (27.3\% vs. $25.0 \%, p=0.973)$ and survival (log-rank test, $p=0.502$ ) were not different between the two groups (Fig. 3B). 
Table 4. Prognostic factors for mortality in pleuroparenchymal fibroelastosis patients assessed using Cox proportional hazard model

\begin{tabular}{|c|c|c|c|c|}
\hline \multirow{2}{*}{ Variable } & \multicolumn{2}{|c|}{ Univariate analysis } & \multicolumn{2}{|c|}{ Multivariate analysis } \\
\hline & $\mathrm{HR}(95 \% \mathrm{CI})$ & $p$ value & $\mathrm{HR}(95 \% \mathrm{CI})$ & $p$ value \\
\hline Age & $1.095(0.937-1.280)$ & 0.253 & & \\
\hline Ever smokers & $1.485(0.134-16.510)$ & 0.748 & & \\
\hline BMI & $0.136(0.016-1.186)$ & 0.071 & & \\
\hline Pneumothorax & $0.004(0.00-358.7)$ & 0.347 & & \\
\hline CRP & $1.675(1.021-2.750)$ & 0.041 & & \\
\hline FVC & $0.894(0.804-0.994)$ & 0.039 & $0.894(0.809-0.988)$ & 0.028 \\
\hline DLco & $0.982(0.933-1.034)$ & 0.496 & & \\
\hline TLC & $0.994(0.922-1.072)$ & 0.886 & & \\
\hline RV & $1.032(0.970-1.097)$ & 0.325 & & \\
\hline 6MWT, distance & $0.980(0.961-0.999)$ & 0.042 & & \\
\hline 6MWT, initial $\mathrm{SpO}_{2}$ & $1.659(0.640-4.299)$ & 0.298 & & \\
\hline 6MWT, lowest $\mathrm{SpO}_{2}$ & $2.905(0.768-0.978)$ & 0.116 & & \\
\hline $\mathrm{PaO}_{2}$ & $1.003(0.959-1.048)$ & 0.900 & & \\
\hline $\mathrm{PaCO}_{2}$ & $0.984(0.678-1.429)$ & 0.934 & & \\
\hline RVSP & $0.881(0.726-1.069)$ & 0.199 & & \\
\hline BAL, WBC count & $1.000(0.998-1.003)$ & 0.742 & & \\
\hline BAL, neutrophil & $1.035(0.983-1.089)$ & 0.189 & & \\
\hline BAL, lymphocyte & $0.974(0.908-1.046)$ & 0.474 & & \\
\hline
\end{tabular}

RV/TLC was not converged due to the small sample size.

HR, hazard ratio; CI, confidence interval; BMI, body mass index; CRP, C-reactive protein; FVC, forced vital capacity; DLco, diffusing capacity of the lung for carbon monoxide; TLC, total lung capacity; RV, residual volume; 6MWT, six-minute walk test; $\mathrm{SpO}_{2}$, saturation of peripheral oxygen; $\mathrm{PaO}_{2}$, partial pressure of oxygen; $\mathrm{PaCO}_{2}$, partial pressure of carbon dioxide; RVSP, right ventricular systolic pressure; BAL, bronchoalveolar lavage; WBC, white blood cell.

\section{DISCUSSION}

In our study, the clinical characteristics of Korean patients with PPFE were similar to those reported previously. Most patients showed a low body mass index (BMI), restrictive lung function, and frequent pneumothorax during follow-up. However, lower lobe involvement was frequently observed; patients with lower lung involvement were older and had a poorer exercise capacity compared with those without lower lung involvement. FVC was a significant prognostic factor in Korean patients with PPFE.

In previous studies, relatively lower BMI and higher residual volume (RV)/TLC in PPFE patients than in other IIP patients were reported as typical features of PPFE $[9,16,17,19,20]$; our results support these findings.
Tanizawa et al. [21] reported that, in 118 patients with fibrotic ILD who registered for lung transplantation, BMI was significantly different between the radiologically defined PPFE and no PPFE groups $\left(15.9 \mathrm{~kg} / \mathrm{m}^{2}\right.$ vs. $21.5 \mathrm{~kg} / \mathrm{m}^{2}, p<0.001$ ). Oda et al. [16], in 108 IPF patients, reported that the combined PPFE group showed lower BMI $\left(18.6 \pm 1.8 \mathrm{~kg} / \mathrm{m}^{2}\right.$ vs. $\left.25.1 \pm 3.6 \mathrm{~kg} / \mathrm{m}^{2}, p<0.01\right)$ and higher RV/TLC ( $42.6 \% \pm 8.7 \%$ vs. $33.1 \% \pm 7.6 \%, p<0.01$ ) than the IPF only group. The reason for lower BMI in patients with PPFE than in other IIP patients is not well known; however, Suzuki et al. [22], on comparing body composition changes between 43 PPFE and 131 IPF patients, suggested that the characteristics of upper lobe predominant fibrosis and more severe restrictive defects in patients with PPFE might exacerbate the impaired energy and protein balance, resulting in weight 
Table 5. Comparison of baseline characteristics between pleuroparenchymal fibroelastosis patients with and without lower lobe involvement

\begin{tabular}{lccc}
\hline Variable & $\begin{array}{c}\text { Lower lobe } \\
(+)\end{array}$ & $\begin{array}{c}\text { Lower lobe } \\
(-)\end{array}$ & p value $^{\mathrm{a}}$ \\
\hline No. of patients & 22 & 4 & \\
Age, yr & $65.4 \pm 7.7$ & $47.0 \pm 5.2$ & 0.001 \\
\hline Male sex & $13(59.1)$ & $3(75.0)$ & 0.656 \\
\hline Ever smokers & $9(40.9)$ & $4(100)$ & 0.069 \\
\hline BMI, kg/m ${ }^{2}$ & $20.0 \pm 3.0$ & $20.6 \pm 2.1$ & 0.656 \\
\hline Pulmonary function & & & \\
\hline FVC, \% predicted & $60.1 \pm 18.8$ & $70.8 \pm 23.6$ & 0.331 \\
\hline DLco, \% predicted & $54.5 \pm 18.7$ & $59.0 \pm 19.1$ & 0.525 \\
\hline TLC, \% predicted & $67.9 \pm 11.9$ & $83.7 \pm 15.6$ & 0.101 \\
\hline RV, \% predicted & $67.0 \pm 19.4$ & $84.7 \pm 9.1$ & 0.185 \\
\hline RV/TLC, \% & $39.3 \pm 9.7$ & $32.3 \pm 5.1$ & 0.185 \\
\hline Six-minute walk test & & & \\
\hline Distance, meter & $428.3 \pm 101.2$ & $567.7 \pm 62.4$ & 0.011 \\
\hline Initial SpO,$\%$ & $96.9 \pm 1.3$ & $97.3 \pm 1.5$ & 0.805 \\
\hline Lowest SpO,$\%$ & $93.8 \pm 3.0$ & $94.7 \pm 3.5$ & 0.742 \\
\hline BAL fluid analysis & & & \\
\hline Neutrophil, \% & $13.7 \pm 15.9$ & $20.3 \pm 31.8$ & 0.935 \\
\hline Lymphocyte, \% & $17.3 \pm 15.9$ & $11.3 \pm 3.2$ & 0.805 \\
\hline Values are presented as & &
\end{tabular}

Values are presented as mean $\pm \mathrm{SD}$ or number (\%). BMI, body mass index; FVC, forced vital capacity; DLco, diffusing capacity of the lung for carbon monoxide; TLC, total lung capacity; RV, residual volume; $\mathrm{SpO}_{2}$, saturation of peripheral oxygen; BAL, bronchoalveolar lavage.

${ }^{a}$ Mann-Whitney's U test or Fisher's exact test.

loss and muscle wasting. In terms of RV/TLC, Oda et al. [16] suggested that, despite upper lobe volume loss, RV/TLC was higher in PPFE patients than in other IIP patients because RV was relatively more increased than TLC owing to compensatory hyperinflation of the middle and lower lobes.

PPFE could be related to relevant underlying conditions including infection, drugs, autoimmune disease, and organ transplantation, and the reports of PPFE after organ transplantation have increased [23-26]. In our study, among 26 patients, two patients (7.7\%) had a history of organ transplantation (one heart and one kidney), consistent with previous results; in the study including 12 patients with PPFE, Reddy et al. [3] reported that one patient (8.3\%) had a history of kidney transplantation.
Cha et al. [12] also reported that among seven Korean patients with PPFE, three patients (42.9\%) had a history of organ transplantation (two bone marrow and one liver).

In patients with PPFE, the coexistence of other ILDs in the lower lobes has been increasingly reported [3,16,27], and the prevalence of coexistent ILD is relatively high ( $43 \%$ to $89 \%$ ), especially in Japanese reports $[9,17]$. In a study including patients with PPFE from western countries, Reddy et al. [3] reported that among 12 patients with PPFE, lower lobe involvement was identified in 50\% of the subjects. Our study also confirmed the high prevalence $(84.6 \%)$ of lower lobe involvement reported by in the Japanese studies. In our study, PPFE patients with lower lobe involvement were older and had a poorer exercise capacity compared with those without lower lobe involvement; however, survival did not differ between the two groups. In a recent study of 40 Japanese patients with clinically diagnosed PPFE, Kono et al. [28] showed results similar to those of our study. PPFE patients with lower lobe involvement $(\mathrm{n}=21$ ) were significantly older $(74.3 \pm 9.3$ years vs. $60.2 \pm 9.9$ years, $p=0.002)$ and had lower lung function including RV $(99.2 \% \pm 27.4 \%$ vs. $130.7 \% \pm 27.8 \%, p=0.03)$ and TLC $(72.3 \% \pm 13.7 \%$ vs. $93.5 \%$ $\pm 16.7 \%, p=0.007)$ than those without lower lobe involvement $(n=19)$. However, survival was significantly worse in PPFE patients with lower lobe involvement than in those without lower lobe involvement (log-rank test, $p$ $=0.014)[28]$.

In our study, among patients with lower lobe involvement, EFs were detected in the lower lobes of one-third of patients (38.5\%). Nakatani et al. [4] also reported that, among 12 patients with PPFE, fibroelastosis was observed in the lower lobe in two patients (16.7\%). In addition, one study comparing the amount of lung EFs $(\mathrm{EF})$ in PPFE patients $(\mathrm{n}=6)$ and in IPF patients $(n=28)$ found that PPFE patients presented significantly more EFs in the lower lobe (EF scores, $23.6 \% \pm 2.4 \%$ vs. $12.2 \% \pm$ $4.4 \%, p<0.0001$ ) [29]. These results suggest that, in some patients, lower lobe involvement could be due to PPFE progression rather than due to the coexistence of other types of ILD.

Our study showed that only FVC was a significant predictive factor for mortality in patients with PPFE. The results of a previous report support our findings: Shioya et al. [30], in a study of 29 PPFE patients, showed that FVC (HR, $0.093 ; 95 \%$ CI, 0.083 to $0.982 ; p=0.017$ ) was an 
independent factor predicting mortality, along with sex, age, and physiology (GAP) index (HR, 2.510; 95\% CI, 1.245 to $5.059 ; p=0.010$ ), in a multivariate Cox proportional hazard model. Previous studies have also reported other prognostic factors in PPFE patients [17,19,31]. Kato el al. [19] reported that, in 36 Japanese patients with PPFE, the UIP pattern in the lower lobe on HRCT was a significant risk factor for poor prognosis (odds ratio, 23.670; 95\% CI, 1.505 to $372.268 ; p=0.024$ ) after adjusting for Krebs von den Lunen-6, modified Medical Research Council, and fibrosis scores. Additionally, Khiroya et al. [31] reported that, in 43 Japanese patients with PPFE, male sex was an independent predictor of mortality (HR, 5.31; 95\% CI, 1.08 to $26.12 ; p=0.04$ ) after adjusting for age.

In our study, most patients were treated with anti-inflammatory or antifibrotic agents, but no differences in survival were observed between patient groups. At present, no effective therapy exists for patients with PPFE. There are only some case reports showing that pirfenidone, an antifibrotic agent used for IPF, reduced the decline in lung function in PPFE patients [32,33]. In patients with PPFE, a UIP pattern in lower lobe involvement is common; therefore, pirfenidone might be useful for PPFE patients with a coexistent UIP pattern in their lower lobes. In the near future, large-scale studies are needed to develop further effective treatments.

This study has some limitations. First, this was a retrospective study conducted in a single center with a relatively small number of patients and short follow-up period. However, the baseline characteristics of our subjects were similar to those of patients in previous reports. Second, pathologic diagnosis was performed using transbronchial lung biopsy in around half (42.3\%) of the subjects. However, a previous study suggested that patients with a clinical diagnosis of PPFE had similar characteristics to those with histopathologically confirmed PPFE patients [9], and in our study, the diagnosis of PPFE was confirmed by multidisciplinary discussion using clinical radiologic and pathologic data. Third, the number of patients without lower lobe involvement was small ( $\mathrm{n}=4,15.4 \%)$, and follow-up duration was short (mean 7.3 months), which could reduce statistical power when comparing patients with and without lower lobe involvement. However, despite the small sample size, patients with lower lobe involvement were characterized by older age and poorer exercise capacity, compared with those without lower lobe involvement.

In conclusion, Korean patients with PPFE showed clinical characteristics and prognoses similar to those reported previously; however, lower lobe involvement was frequently observed. Patients with lower lung involvement were older and had a poorer exercise capacity compared with those without lower lung involvement.

\section{KEY MESSAGE}

1. Clinico-radiologic-pathologic features and prognosis in Korean patients with pleuroparenchymal fibroelastosis (PPFE) are similar to those reported previously.

2. Lower lobe involvement on high-resolution computed tomography is frequently observed in Korean patients with PPFE.

3. PPFE patients with lower lung involvement are characterized by older age, and poorer exercise capacity compared with those without lower lung involvement.

\section{Conflict of interest}

No potential conflict of interest relevant to this article was reported.

\section{Acknowledgments}

This work was supported by a grant from the Basic Science Research Program through the National Research Foundation of Korea (NRF), which is funded by the Ministry of Science and Technology (NRF2019R1A2C2008541).

\section{REFERENCES}

1. Travis WD, Costabel U, Hansell DM, et al. An official American Thoracic Society/European Respiratory Society statement: update of the international multidisciplinary classification of the idiopathic interstitial pneumonias. Am J Respir Crit Care Med 2013;188:733-748.

2. Frankel SK, Cool CD, Lynch DA, Brown KK. Idiopathic pleuroparenchymal fibroelastosis: description of a novel clinicopathologic entity. Chest 2004;126:2007-2013.

3. Reddy TL, Tominaga M, Hansell DM, et al. Pleuroparen- 
chymal fibroelastosis: a spectrum of histopathological and imaging phenotypes. Eur Respir J 2012;40:377-385.

4. Nakatani T, Arai T, Kitaichi M, et al. Pleuroparenchymal fibroelastosis from a consecutive database: a rare disease entity? Eur Respir J 2015;45:1183-1186.

5. Watanabe K, Nagata N, Kitasato Y, et al. Rapid decrease in forced vital capacity in patients with idiopathic pulmonary upper lobe fibrosis. Respir Investig 2012;50:88-97.

6. Watanabe K. Pleuroparenchymal fibroelastosis: its clinical characteristics. Curr Respir Med Rev 2013;9:299-237.

7. von der Thusen JH. Pleuroparenchymal fibroelastosis: its pathological characteristics. Curr Respir Med Rev 2013;9:238-247.

8. Yoshida Y, Nagata N, Tsuruta N, et al. Heterogeneous clinical features in patients with pulmonary fibrosis showing histology of pleuroparenchymal fibroelastosis. Respir Investig 2016;54:162-169.

9. Enomoto Y, Nakamura Y, Satake Y, et al. Clinical diagnosis of idiopathic pleuroparenchymal fibroelastosis: a retrospective multicenter study. Respir Med 2017;133:1-5.

10. Park CS, Jeon JW, Park SW, et al. Nonspecific interstitial pneumonia/fibrosis: clinical manifestations, histologic and radiologic features. Korean J Intern Med 1996;11:122132.

11. Lim GI, Lee KH, Jeong SW, et al. Clinical features of interstitial lung diseases. Korean J Intern Med 1996;11:113121.

12. Cha YJ, Han J, Chung MP, Kim TJ, Shin S. Pleuroparenchymal fibroelastosis in heterogeneous clinical conditions: clinicopathologic analysis of 7 cases. Clin Respir J 2018;12:1495-1502.

13. Wanger J, Clausen JL, Coates A, et al. Standardisation of the measurement of lung volumes. Eur Respir J 2005;26:511522.

14. Miller MR, Hankinson J, Brusasco V, et al. Standardisation of spirometry. Eur Respir J 2005;26:319-338.

15. Macintyre N, Crapo RO, Viegi G, et al. Standardisation of the single-breath determination of carbon monoxide uptake in the lung. Eur Respir J 2005;26:720-735.

16. Oda T, Ogura T, Kitamura H, et al. Distinct characteristics of pleuroparenchymal fibroelastosis with usual interstitial pneumonia compared with idiopathic pulmonary fibrosis. Chest 2014;146:1248-1255.

17. Ishii H, Watanabe K, Kushima H, et al. Pleuroparenchymal fibroelastosis diagnosed by multidisciplinary discussions in Japan. Respir Med 2018;141:190-197.
18. Holland AE, Spruit MA, Troosters T, et al. An official European Respiratory Society/American Thoracic Society technical standard: field walking tests in chronic respiratory disease. Eur Respir J 2014;44:1428-1446.

19. Kato M, Sasaki S, Kurokawa K, et al. Usual interstitial pneumonia pattern in the lower lung lobes as a prognostic factor in idiopathic pleuroparenchymal fibroelastosis. Respiration 2019;97:319-328.

20. Kusagaya H, Nakamura Y, Kono M, et al. Idiopathic pleuroparenchymal fibroelastosis: consideration of a clinicopathological entity in a series of Japanese patients. BMC Pulm Med 2012;12:72.

21. Tanizawa K, Handa T, Kubo T, et al. Clinical significance of radiological pleuroparenchymal fibroelastosis pattern in interstitial lung disease patients registered for lung transplantation: a retrospective cohort study. Respir Res 2018;19:162.

22. Suzuki Y, Yoshimura K, Enomoto Y, et al. Distinct profile and prognostic impact of body composition changes in idiopathic pulmonary fibrosis and idiopathic pleuroparenchymal fibroelastosis. Sci Rep 2018;8:14074.

23. Mariani F, Gatti B, Rocca A, et al. Pleuroparenchymal fibroelastosis: the prevalence of secondary forms in hematopoietic stem cell and lung transplantation recipients. Diagn Interv Radiol 2016;22:400-406.

24. Takeuchi Y, Miyagawa-Hayashino A, Chen F, et al. Pleuroparenchymal fibroelastosis and non-specific interstitial pneumonia: frequent pulmonary sequelae of haematopoietic stem cell transplantation. Histopathology 2015;66:536-544.

25. Ishii T, Bandoh S, Kanaji N, et al. Air-leak syndrome by pleuroparenchymal fibroelastosis after bone marrow transplantation. Intern Med 2016;55:105-111.

26. Kinoshita Y, Watanabe K, Ishii H, et al. Pleuroparenchymal fibroelastosis as a histological background of autoimmune diseases. Virchows Arch 2019;474:97-104.

27. Esteves C, Costa FR, Redondo MT, et al. Pleuroparenchymal fibroelastosis: role of high-resolution computed tomography (HRCT) and CT-guided transthoracic core lung biopsy. Insights Imaging 2016;7:155-162.

28. Kono M, Fujita Y, Takeda K, et al. Clinical significance of lower-lobe interstitial lung disease on high-resolution computed tomography in patients with idiopathic pleuroparenchymal fibroelastosis. Respir Med 2019;154:122-126.

29. Enomoto N, Kusagaya H, Oyama Y, et al. Quantitative analysis of lung elastic fibers in idiopathic pleuroparen- 
chymal fibroelastosis (IPPFE): comparison of clinical, radiological, and pathological findings with those of idiopathic pulmonary fibrosis (IPF). BMC Pulm Med 2014;14:91.

30. Shioya M, Otsuka M, Yamada G, et al. Poorer prognosis of idiopathic pleuroparenchymal fibroelastosis compared with idiopathic pulmonary fibrosis in advanced stage. Can Respir J 2018;2018:6043053.

31. Khiroya R, Macaluso C, Montero MA, et al. Pleuroparenchymal fibroelastosis: a review of histopathologic features and the relationship between histologic parameters and survival. Am J Surg Pathol 2017;41:1683-1689.

32. Sato S, Hanibuchi M, Takahashi M, et al. A patient with idiopathic pleuroparenchymal fibroelastosis showing a sustained pulmonary function due to treatment with pirfenidone. Intern Med 2016;55:497-501.

33. Boerner EB, Costabel U, Wessendorf TE, Theegarten D, Bonella F. Idiopathic pleuroparenchymal fibroelastosis (PPFE): a case study of a rare entity. Rev Port Pneumol (2006) 2017;23:352-355. 